allerdings noch dutzende von versen, die nach meinem gefühl bieher gehören, nämlich die von der form

und vor allem

Ic eom Hrốzáres (335)

pæt hie sint wilcuman (388).

Ich kann die zerschneidung der composita als rhythmisches princip am versschluss so wenig naturlich finden als am anfang (etwa 2282 frioðowáre bced) und sehe keinen zwingenden grund, pat hie in béorsele (482) anders zu lesen als pat hine on ylde (22, s. Beitr. 10, 284). Ich kann den gegensatz, in welchen bei Sievers' betonungsweise die zwei compositionsglieder treten, nur verstehen, wenn sie die zwei wesentlichen bestandteile des verses siud, wie etwa in $\mathbf{\nabla} .771$ pot se winsele, 801 pone synscaðan. Sonst halte ich die betonung wie sie z. b. im Nibelungenlied an der Bartsch'schen stelle sich so oft findet (hêrlicher), d. b. unnittelbare unterordnung des zweiten gliedes unter das erste, für geboten. In versen wie wisle pám áhlácan (646) kann wiste schwächer betont sein als láe und doch bebung, sobald es nur in seiner region vorherscht und lác in seiner - der der höchsten erhebung des verses - untergeordnet ist. Doch ich kann nicht hoffen ohne sehr weit auszuholen meine auffassung der von Sievers gegentiber zu rechtfertigen und muss deshalb fir diesmal darauf verzichten, dem zweiten halbvers eine ähnliche stilistische heweglichkeit des hauptstabes nachzuweisen wie dem ersten.

WÜRZBURG, september 1894 . $\quad$ O. BRENNER.

\title{
ZUM RHYTHMUS DER NIBELUNGEN- UND GUDRUNSTROPHEN.
}

Dass eine rhythmische reibe von verschiedenen verschieden aufgefasst, infolgedessen verschieden vorgetragen und nachgebildet werden kann ist einleuchtend und jungst von Sievers an der ïberlieferung der Marienlieder schön nachgewiesen. Auch an den Nibelungen zeigt sich ein wandel der rhythmischen 
auffassung des uberlieferten. Heusler hat zu zeigen versucht, dass die letzte halbzeile einst durchweg nur drei hebungen gehabt habe und dass die späteren bearbeiter eine vierte hinzugesetzt bätten. Ich kann mir nicht vorstellen, wie in einer naiven entwicklungsperiode jemand dazu gekommen wäre, einer dipodischen reihe einen einzelnen versfuss anzuflicken; der versfuss ist kein selbständiger teil, den man etwa als kehrreim ablösen und ein- und anftigen konnte. Erst als man lateinische und französische kunstdichtungen von podischem bau mit bald 4 , bald 5, 6, 7 hebigen versen zu vorbildern uahm, lernte man einzelne füsse als selbständige versglieder beliebig an- und abstossen. Selbst die Gudrunstrophe ist nicht aus freier operation mit einzelfuissen entstanden. Richtig ist, dass die Nibelungenstrophe ehedem in der letzten balbzeile sehr oft nur drei füsse (oinen ganzen und einen verkurzten takt $\dot{x} \times \times \times \mid{ }^{\prime}$ ), wol meist nit auftakt hatte; richtig aber auch, dass viele jetzt als 4 hebig angesehene verse eine verkurzung, zurluckfuhrung auf eine ( $5-6$ silbige) dreibebige grundform $\mathrm{n}$ i $\mathrm{c}$ h t vertragen. Giengen drei- und vierhebige verse nebeneinander ber? Kaum. Versen wie ine gesach sô gerne hie und dô wir schieden von dan sieht man nicht mit bestimmtheit an, ob sie der 8. oder einer andern graden balbzeile angehören. Der eine wird sie vier-, der andere dreikebig lesen. Itnen glichen ehedem die allermeisten langen schlussżeileu, d. h. starker, zwei- bis dreisilbiger auftakt wurde zur hervorluebung der letzten halbzeile regel. ${ }^{1}$ ) In den volkstumlichen vierzeilern der gegenwart lässt sich dieselbe neigung zur verstärkung des auftaktes beobachten. Solange der dipodische vortrag allgemein und fest war, wurden verse der obigen gattung als $\times \times \times|\dot{x} \times \times \times| \dot{x}$ aufgefasst und nachgebildet; sobald die romanische vortragsweise auch auf die einheimischen gebilde libertragen wurde, erhielt der stetige

1) Paul, Grundr. 2, 1, 932 bezeichnet den letzten halbvers als typus B, dann wäre halbz. 8 von anfang an von $6,4,2$ verschieden, die wol als D-typus aufzufassen sind. Ich halte freilich die anwendung der typen auf die späten reimverse vom standpunkt der typentheorie aus für gewagt, da es scheinen könnte, dass die typen, wenn sie anf zwei so sehr verschiedene versgattungen passen, dem we sen beider nicht gerecht werden - oder dass die verschiedenheit nicht so gross ist als die typentheorie voraussetzt. 
auftakt seine selbständigkeit als besonderer fuss, und in den nachbildungen traten auch stark betonte silben an die stelle des alten auftaktes. Dass die 8. halbzeile nicht von anfang an vierbebig gedacht war, ist leicht zu ersehen, wenn man sie mit einer bewusst vierhebig gebauten nachbildung vergleicht, der ubersetzung Hinsbergs (München 1812, proben in Hellinghaus' Nibelungenlied nach den besten ubersetzern), z. b. den versen

\section{ergrimme könig Etzels weib \\ frauen und jungfrauen schwer \\ und schlichtete der helden streit}

etc. etc. Eine umständliche untersuchung uber die stellung der eigennamen in der $8 . \mathrm{bz}^{1}$ ), die ich hier nicht vorzuf(ibren brauche, hat mir bestätigt, dass die erste hebung ursprlinglich die ist, die man gewöhulich als zweite zählt, dass die erweiterung am anfang, nicht in der mitte der halbzeile stattgefunden hat.

Der bearbeiter von $\mathrm{C}$ hat die Nibelungenverse nicht dipodisch gelesen und hat den letzten auftakt schon als selbstständigen fuss behandelt: das wird sich jedem leser aufdrängen. Bekanntlich hat Bartsch darauf aufmerksam gemacht, dass in $\mathrm{C}$ die plusstroplen im letzten halbvers nicht die vertretung des 2. (eigentlich 1.) fusses durch eine länge kennen. Bartsch hat damit nur ein symptom genannt. $\mathrm{C}$ hat die läugen deshalb nicht, weil seive auffassung des verses sie nicht bedingte. Die länge überhaupt (eigentlich uberlänge, s. darluber meine ausfuhrung in der 'Festschrift' furr R. Hildebrand) ist aus dem dipodischem bau herausgewachsen, ein mittel, um der haupthebung ihr starkes gewicht zu sichern und an der Bartsch'schen stelle insbesondere, um das verhältnis zu den vorausgehenden auftaktsilben (der eingangssenkung) klar herauszuarbeiten. Unumgänglich notwendig war die länge als fussfüllung nicht, deshalb darf ibr fehlen nicht schlechthin als kriterium für die 'unechtheit' verwendet werden, sondern nur dann, wenn das fehlen aus dem podischen bau sich erklärt. Ebenso wird auch ubberlänge in der reimsilbe klingender verse und vor der cäsur als ergebnis des dipodischen baues anzuerkennen sein:

1) Vgl. 6 die Guntheres man, 2 zuo Guntheres man mit 8 des künec G.'s man öfter, alle Guntheres man, genuoge G.'s man. 
der ausgang " $\check{x}$ wird hier dem versrhythmus gerecht.1) Mit dem eindringen podischer messung wird die verstärkung der vorletzten hebung uberflussig, unnatülich und ungewohnt. So ist es kein zufall, dass seit dem gesteigerten einfluss der höfischen dichtung der Romanen (die volkstumliche kennt auch bei ihnen dipodische verse mit dem ausgang " $\check{X}^{2}$ ) die vierhebigen klingenden verse zunehmen (s. dartuber nun A. Heusler, Ueber germ. versbau s. $67 \mathrm{ff}$.). Undeutsch sind sie aber von anfang an nicht, das hat m. e. Heusler erwiesen.

So glaube ich nun allerdings auch jetzt noch, dass die Gudrunverse von der neuen vortragsweise beeinflusst sind. Die wortfullung ist nicht mehr mit dem ausgeprägten rbythmischen gefuhl gewählt wie in den älteren Nibelungeustrophen. Aber der rhythmus ist, was ich fruther bezweifelte, doch noch dipodisch. Eine andere frage ist, ob die ausgänge $-x$ der 1., 3., 5-8. balbzeilen einen oder zwei fusse darstellen. Ich hoffe sie etwas bestimmter beantworten zu können als fruher. Die weit verbreitete vorliebe für die zusammenstellung klingender căsur und stumpfen versschlusses (die umgekehrte ordnung ist seltener) ist wol nicht zufällig. Der ausgang $\stackrel{\sim}{ } \dot{x}$, bei dem die stimme zuletzt noch in einiger höhe (stärke) bleibt, entspricht der betonung im satzinvern: zur vollendung des satzes ist die wogende betonung nicht geeeignet; ì éntgègèn fălllt die stinime oder sie steigt gleichmässig empor. Ich verweise auf das oben uber den ursprünglichen charakter der zweiten hälfte der stabreimzeile gesagte. Da nun die 5. und 7. halbzeile der Gudrunstrophe in weitaus den meisten fällen mitten im satze schliessen, wird hier der ausgang $>$ d. i. "゙்́ anzunehmen sein; und da die 8. so gut wie ausnahmslos mit starkem sinneseinschnitt ausgebt, wird hier d. i. $\perp \dot{x}$ am platze sein. Die 7. halbzeile wird aber nicht einfach von der 8 . bestimmt, sondern sie schliesst selbst auffällig oft (in den ersten 50 strophen z. b. 32 mal!) mit einem sinnesabschluss. So ist das schema der 3. und 4. zeile der Gudrun:

1) Er entspricht selbst einem dipodischen takt $|\ddot{x} \times \dot{x} \times|$, nur ist die letzte more durch pause vertreten.

2) Franz. beispiele aus älterer und neuer zeit vor allem bei 'Tiersot, Chans. pop. en France. 


$$
\begin{aligned}
& \text { 3. } \times|\ddot{x} \times \dot{x} \times| " \ddot{x} \wedge|: \ddot{x} \times \dot{x} \times| \stackrel{-}{x} \wedge \wedge
\end{aligned}
$$

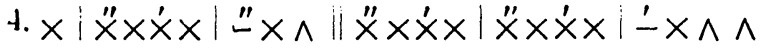

Ob nun in der Nibelungen- und Gudrunstrophe der klingende schluss in der cäsur einfussig wurde oder zweifussig blieb wage ich nicht zu entscheiden. Nach der ältesten messung war sicher der ausgang $" \dot{X}$, in den jungeren, vor allem bei Nibel. C, wäre $\dot{-} \dot{x}$, zumal vor starker interpunction, nicht undenkbar; es ist aber zu beachten, dass der untersclied sebr gering ist: das tonverhältnis beider silben bleibt gleich $":,=,:$, das quantitätsverhältnis wenigstens älınlich, denn da auch bei einfussiger messung der letzten silbe eine pause folgt, so kann der ausgang $\dot{-} \dot{x}$ oder seine länge - unbeschadet des gesammtrhy thmus etwas uber das mass eines gewölınlichen fusses (oder seiner hebung) gedehut worden sein. Vor stärkerer interpunction lag die einfüssige messung näher als mitten im satz; bei ausgesprochen dipodischem vortrag wider mag sich, wie oben gesagt, die zweifüssige besser erhalten haben.

Wie sollen nun wir, wie soll man in der schule die verse der Nibelungen und der Gudrun lesen? Soweit der dipodische charakter deutlich zu tage tritt, naturlich dipodisch. Wo er nur leicht verdeckt ist, wird an vielen stellen die kritik die mittel liefern, ihn herzustellen. Bei manchen wird widerum dem rhythmus und der naturlichen betonung dadurch rechnung getragen werden können, dass man zur hervorhebung neben der gesteigerten exspiration auch tonerhöhung verwendet. Den rest in das dipodische schema zwängen zu wollen wird sich böchstens in der schule empfehlen, wo man das grundschema nicht verlieren will, sonst wird man den verschiedenen bearbeitungsschichten ihr recht lassen mlissen und also einen 8 . halbvers wie ze jungest an den werken lit (Hzm. 274) wirklich vierhebig zu lesen baben.

Es liegt nahe bei den kurzzeilen des höfischen epos die gleichen fragen zu stellen. Henrici erklärt in seiner Iweinausgabe kurzweg: Hartmann kenne keine dipodische messung. Man sollte meinen, verse wie

$$
\begin{aligned}
& \text { verwieset zewâre } \\
& \text { mit spannelangeme hâre } \\
& \text { breit alsam ein wanne } \\
& \text { dem ungeviegen manne }
\end{aligned}
$$


(Iw. 441-44), ja die allermeisten klingenden milssten unmittelbar, obne theoretische erwägungen als dipodisch erkannt werden, und die stumpfen wie

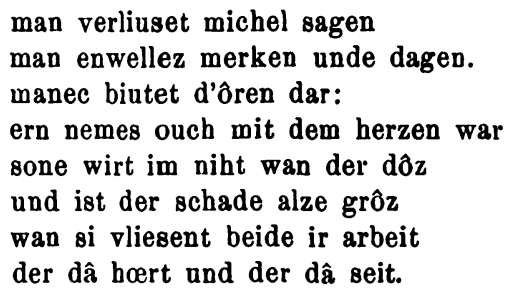

(Iw. 249-56) scheinen in dieser aufeinanderfolge in beabsichtigtem dipodischem rhythmus dahin zu fliessen (bald ist die erste, bald die zweite hebung der takte der giffel). Sicher ist daneben ein grosser teil der stumpfen verse nur podisch zu lesen. Diese mischung wird fortbestanden haben, solange cs sogen. dreihebig-klingende verse gab. Gerade der zweifussige klingende reim ist, wie auch Siever's (Forschungen, Festgabe f. R. Hildebrand 26) hervorhebt, charakteristisch fur die dipodische messung. Freilich so ungemischt dipodisch wie Wernhers lieder sind die dichtungen der höfischen epiker nicht. R. M. Meyers untersuchungen dürten in dieser richtung jetzt eine ergänzung brauchen.

. . . WÜRZBURG, . september. 1894.

O. BRENNER. 